\title{
Expression and purification of the antimicrobial peptide CM4 in Escherichia coli
}

\author{
Liangfan Zhou • Qingping Lin · Baocun Li • \\ Nannan Li $\cdot$ Shuangquan Zhang
}

Published online: 25 February 2009

(C) Springer Science+Business Media B.V. 2009

\section{Erratum to: Biotechnol Lett (2009) 31:437-441 DOI 10.1007/s10529-008-9893-0}

The results given in Figs. 3 and 4 and Table 2 of the original publication were partly incorrect or inaccurate (notably the peak in Fig. 3, the corresponding retention time specified in the text, the missing information on the thickness of the medium and the diameter of the pore in Fig. 4 and some data in Table 2). The corrected Figs. 3 and 4 and Table 2 are shown below.

The online version of the original article can be found under doi:10.1007/s10529-008-9893-0.

L. Zhou · Q. Lin · B. Li · N. Li · S. Zhang $(\varangle)$ Jiangsu Province Key Laboratory for Molecular and Medical Biotechnology, Life Sciences College, Nanjing Normal University, Nanjing 210046, China

e-mail: zhangsq1218@yahoo.com.cn

S. Zhang

Jiangsu Key Laboratory for Supermolecular Medicinal Materials and Applications, Life Sciences College, Nanjing Normal University, Nanjing 210046, China

S. Zhang

Jiangsu Engineering Research Center for Biomedical Function Materials, Nanjing Normal University, Nanjing 210046, China

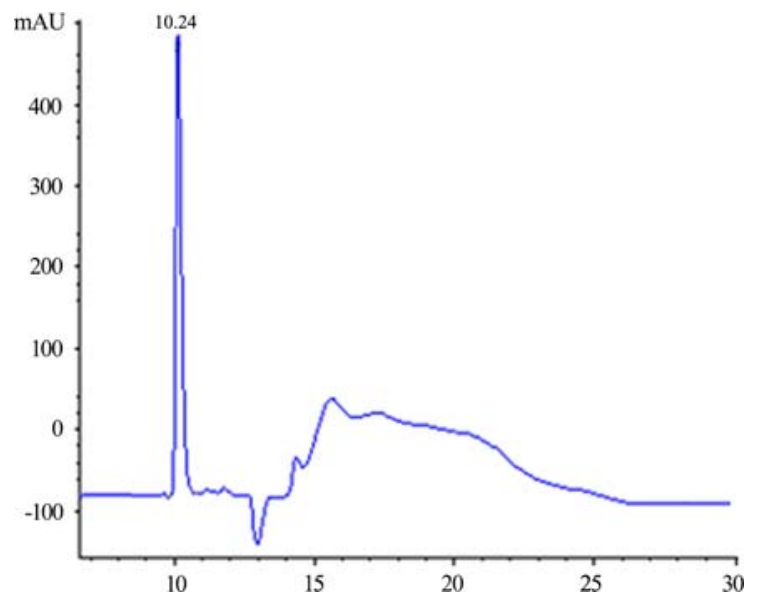

Fig. 3 Purification of recombinant CM4 by RP-HPLC that was performed on HPLC with a Kromasil C18 analytical column $(10 \times 250 \mathrm{~mm}, 5 \mu \mathrm{m}$ bead diameter $)$. The elution was performed using a linear gradient of $15-70 \%$ acetonitrile in $0.1 \%$ trifluoroacetic acid (TFA) at $1.0 \mathrm{ml} / \mathrm{min}$. Elution was monitored at 214 and $280 \mathrm{~nm}$ 


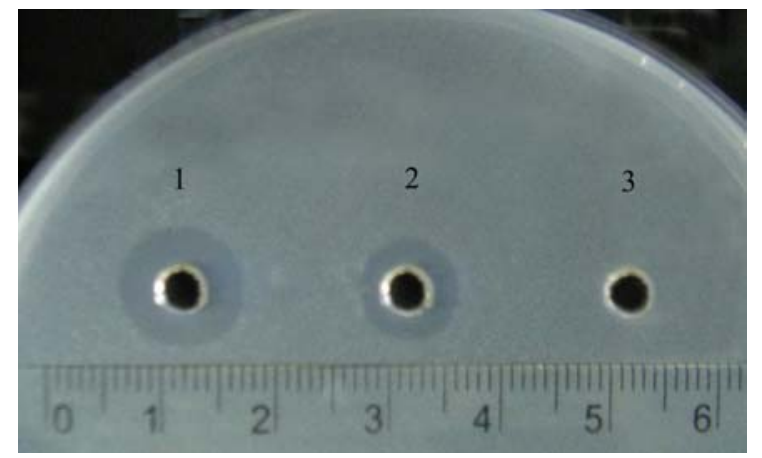

Fig. 4 The bioactivity of CM4 against E. coli $\mathrm{K}_{12} \mathrm{D}_{31}$. The thickness of the medium is $2 \mathrm{~mm}$; the diameter of the pore is $4 \mathrm{~mm}$. (1) The positive control, native CM4 (40 $\mu \mathrm{M})$; (2) recombinant CM4 solution $(20 \mu \mathrm{M})$; (3) the negative control, sterile water
Table 2 Antibacterial activities of recombinant CM4 on selected microorganisms

\begin{tabular}{llc}
\hline Microorganisms $^{\mathrm{a}}$ & \multicolumn{2}{c}{ Minimal inhibitory concentration $(\mu \mathrm{M})$} \\
\cline { 2 - 3 } & Recombinant CM4 & Native CM4 \\
\hline Gram negative & & 8 \\
E. coli K12D31 & 8 & 16 \\
Salmonella spp. & 16 & \\
Fungi & & 16 \\
P. chrysogenum & 16 & 4 \\
A. niger & 4 &
\end{tabular}

${ }^{\text {a }}$ Cells were grown in LB-broth for $24 \mathrm{~h}$ with vigorous shaking at $30^{\circ} \mathrm{C}$

b Stocks solution of CM4 was serially diluted with 0.01 acetic acid and $0.2 \%$ BSA. The antibacterial effect was evaluated by measuring the culture OD600 using a microtiter reader 